\title{
Grading of astrocytomas using a Quantimet 720 image-analysing computer
}

\author{
A. J. ROBERTSON, J. M. ANDERSON, R. A. BROWN, W. SlidDeRS, AND \\ J. SWANSON BECK
}

From the Departments of Pathology and Mathematics, University of Dundee

SUMMARY Cerebral astrocytomas and undifferentiated gliomas were graded by (1) Kernohan classification on the basis of visual microscopy, and (2) optical density/area profiles obtained by automated densitometry with a Quantimet 720 image-analysing computer. In a retrospective study of 44 patients the Quantimet measurements seemed to give a more accurate assessment of prognosis than visual grading.

The histological classification of cerebral neoplasms described by Kernohan and Sayre (1952) remains in popular use, although its value as a prognostic guide is not widely accepted (Russell and Rubinstein, 1977). In practice this classification is mainly applied to astrocytic and poorly differentiated gliomas, which are placed in four arbitrary grades on the basis of cellularity, pleomorphism of nuclei, mitotic activity, vascular proliferation, and necrosis. Kernohan and Sayre (1952) claimed a good correlation between their grading scheme and the survival time of patients, but further studies have failed to confirm this (Weir, 1973; Sherbet and Lakshmi, 1974; Weir and Grace, 1976).

Visual grading is a highly subjective process since it largely depends on the observer's assessment of the characteristics of the microscope image that can be accurately discriminated by the human eye, such as shape, size, and distribution of cells in the section. It takes little account of variations in intensity of staining, which are poorly assessed by the human eye, particularly in non-contiguous areas. Accordingly we have attempted to grade astrocytic gliomas by measuring optical density/area profiles by the approach suggested by Bond (1976) with a Quantimet 720 image-analysing computer. We have shown that this apparatus, which can be used objectively, gives reproducible results that appear to give a better indication of prognosis for the patient than visual grading.

\section{Material and methods}

\section{PATIENTS}

The series was drawn from 65 adult patients indexed in the records of the Pathology Departments of Dundee Royal Infirmary and Ninewells Hospital, Dundee, between 1967 and 1974 as having had cerebral astrocytomas or undifferentiated gliomas. Twenty-one patients were excluded either because they had survived less than two weeks after operation (7), or were lost to follow-up (1), or because the specimen was too small or distorted for Quantimet measurements (13). Observations were made on surgical specimens from the remaining 44 patients $(20$ men and 24 women). At the time of operation their ages ranged from 23 to 70 years (mean 50.8 years).

Surgical treatment consisted of total excision of the neoplasm in five patients, subtotal or partial removal in 25, and biopsy alone in 14. Radiotherapy was given to 15 patients aged 32 to 61 years (mean 46.3 years). The tumour was totally or partially resected in 13 of these 15 patients and biopsy alone was done in two. Follow-up for the purposes of this study was stopped in September 1977. At that time only two patients were alive. Both postoperative survival time and total duration of illness were recorded. The total duration of illness was taken from the time of onset of non-relapsing symptoms or signs of cerebral tumour. In a few cases with a very long history there was doubt about the first sign-as, for example, the development of epilepsy at some distant time. For such patients the shorter course more definitely attributable to brain tumour was used. 


\section{LIGHT MICROSCOPY}

The original sections were reviewed and a Kernohan grading was assigned by one observer (JMA) without knowing the clinical or pathological details. The number of mitotic figures in 20 successive fields under a $\times \mathbf{4 0}$ objective was counted. The average was recorded as the mitotic index.

\section{AUTOMATED HISTOMETRY}

Measurements with the Quantimet 720 imageanalysing computer (Imanco, Cambridge Instruments) were made independently of the visual observations.

The Quantimet 720 system we used consists essentially of a microscope with a television camera attached and a densitometer which can be calibrated to make densitometric measurements over a television monitor field of area $5 \times 10^{5}$ picture points. The densitometer module can be programmed to give sequential measurements over a scale of 64 optical density thresholds from 0 (white) to 63 (black). For histological work only the first 32 thresholds (0-31) are needed. The difference between the number of picture points recorded at successive optical density thresholds is the area of the histological field which lies between these thresholds, and the plot of these 31 areas as a histogram represents the optical density/area profile. Multiple fields can be measured in this way and a mean optical density/ area profile calculated and plotted.

The preparatory histological techniques were standardised; $7 \mu \mathrm{m}$ sections were cut from paraffinembedded blocks of tissue fixed in $4 \%$ neutral buffered formaldehyde and postfixed in formolsublimate; the sections were stained in Mayer's haemalum for five minutes; counterstaining was omitted.

The field measurements were made at ocular magnification $\times 10$ and objective $\times 40$. For each biopsy 20 fields were selected, avoiding areas of necrosis and haemorrhage and non-neoplastic brain tissue, and a mean optical density/area profile was obtained.

A range of optical density profiles was found. In general the histologically less malignant neoplasms contained larger areas stained at lower optical density and smaller areas at higher optical density: the profiles for the more malignant neoplasms showed a 'shift to the right' (Fig. 1). To obtain an index of this 'shift' the optical density/area profile of the neoplasms from each of the $\mathbf{4 4}$ patients was compared with that from a Kernohan grade 1 astrocytoma (from a child not included in this adult series). The index was obtained by taking the difference in the area of the profiles at the thresholds 6 to 31 between each patient and the standard patient and calculating the

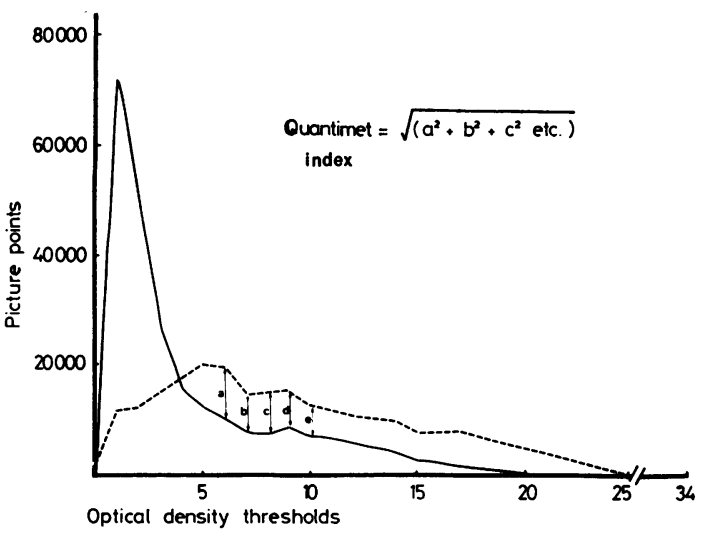

Fig. 1 Diagram showing method of deriving Quantimet index. Solid line is the optical density/area profile of reference patient, a child with a grade 1 astrocytoma, interrupted line is optical density/area profile of patient with a grade 4 astrocytoma. Index obtained by taking the square root of the sum of the squared differences in area above threshold 5.

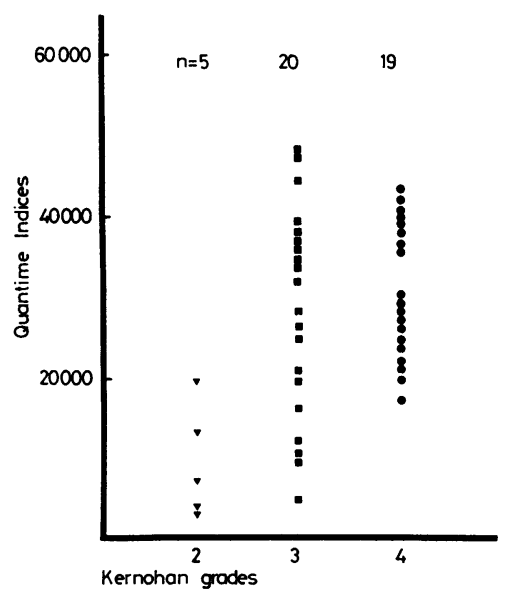

Fig. 2 Scatter diagram showing relationship in a series of astrocytomas between visual grading with the Kernohan method and Quantimet indices.

square root of the sum of the squared differences. The part of the profile below threshold 6 was discarded because of variability of recordings at low optical densities largely attributable to artefacts in the histological sections.

Results

VISUAL GRADING COMPARED WITH QUANTIMET INDICES

In this series of adult patients no grade 1 gliomas 
were encountered. Five were assigned to grade 2, 20 to grade 3 , and 19 to grade 4 . Figure 2 shows the relationship between Quantimet indices and the visual grading. Relatively low indices $(<20000)$ were recorded for all grade 2 astrocytomas, but comparable indices were found for some grade 3 neoplasms. Indices for the remainder of grade 3 fell into the same range as those for grade $4(20000$ 50000 ).

\section{SURVIVAL TIME}

Scrutiny of the mean survival time of patients showed that there were considerable differences between patients given radiotherapy and patients treated by surgery alone (Table 1). Overall, patients given radiotherapy lived longer irrespective of the grade of tumour (with the exception of the total survival time of patients considered to have Kernohan grade 2 tumours). Accordingly, comparisons between visual assessment and Quantimet measurements were made separately on these two groups of patients. Coefficients of correlation are listed in Table 2.

\section{Postoperative survival time}

(a) Patients not receiving radiotherapy Visual grading did not correlate significantly with postoperative survival time. Figure 3 shows that there was little difference in the survival time of patients with grades 3 and 4 neoplasms, apart from one patient with a grade 3 tumour who was still alive five years after operation. The mitotic index, an important part of visual grading, also correlated poorly with postoperative survival time. On the other hand, postoperative survival was significantly correlated

Table 1 Mean survival time of patients grouped by tumour grading

\begin{tabular}{|c|c|c|c|}
\hline \multirow{2}{*}{$\begin{array}{l}\text { Method of } \\
\text { tumour } \\
\text { assessment }\end{array}$} & \multirow{2}{*}{$\begin{array}{l}\text { No. of } \\
\text { patients }\end{array}$} & \multicolumn{2}{|c|}{ Mean survival time (months) } \\
\hline & & Postoperative & Total duration \\
\hline \multicolumn{4}{|l|}{ Visual } \\
\hline Grade 2 & 2 & $20 \cdot 0$ & $59 \cdot 5$ \\
\hline Grade 3 & 14 & $7 \cdot 3$ & $17 \cdot 4$ \\
\hline Grade 4 & 13 & $3 \cdot 0$ & $9 \cdot 2$ \\
\hline \multicolumn{4}{|l|}{ (b) Radiotherapy } \\
\hline Grade 2 & 3 & $32 \cdot 2$ & $45 \cdot 3$ \\
\hline Grade 3 & 6 & $16 \cdot 8$ & $26 \cdot 8$ \\
\hline Grade 4 & 6 & $18 \cdot 3$ & $25 \cdot 3$ \\
\hline \multicolumn{4}{|l|}{$\begin{array}{l}\text { Quantimet } \\
\text { (a) No radiotherapy }\end{array}$} \\
\hline $\begin{array}{l}\text { (a) No radiotherapy } \\
<20000\end{array}$ & 7 & 15.9 & 38.8 \\
\hline $20001-30000$ & 10 & 4.6 & $14 \cdot 2$ \\
\hline $30001-40000$ & 8 & 1.9 & $7 \cdot 0$ \\
\hline$>40000$ & 4 & $2 \cdot 1$ & $3 \cdot 0$ \\
\hline \multicolumn{4}{|l|}{ (b) Radiotherapy } \\
\hline$<20000$ & 5 & $32 \cdot 2$ & $51 \cdot 2$ \\
\hline $20001-30000$ & 3 & $20 \cdot 7$ & $24 \cdot 3$ \\
\hline $30001-40000$ & 5 & 8.9 & $12 \cdot 1$ \\
\hline$>40000$ & 2 & $20 \cdot 0$ & $29 \cdot 5$ \\
\hline
\end{tabular}

Table 2 Coefficients of linear correlation between tumour grading and patients' survival times

\begin{tabular}{|c|c|c|c|c|c|c|}
\hline \multirow{2}{*}{\multicolumn{2}{|c|}{ Tumour grading }} & \multirow[t]{2}{*}{$\begin{array}{l}\text { No. of } \\
\text { patients }\end{array}$} & \multicolumn{2}{|c|}{$\begin{array}{l}\text { Postoperative } \\
\text { survival time }\end{array}$} & \multicolumn{2}{|c|}{$\begin{array}{l}\text { Total duration } \\
\text { of illness }\end{array}$} \\
\hline & & & $r$ & $\mathbf{P}$ & $\boldsymbol{r}$ & $\mathbf{P}$ \\
\hline \multirow[t]{6}{*}{ (a) } & No radiotherapy & & & & & \\
\hline & Visual grading & 29 & 0.34 & ns & 0.43 & $<0.05$ \\
\hline & $\begin{array}{l}\text { Mitotic index } \\
\text { Quantimet indices }\end{array}$ & 29 & $0 \cdot 28$ & ns & $0 \cdot 27$ & ns \\
\hline & 1 All values & 29 & 0.50 & $<0.01$ & 0.59 & $<0.001$ \\
\hline & 2 Values $>10000$ & 25 & 0.43 & $<0.05$ & 0.48 & $<0.05$ \\
\hline & 3 Values > 15000 & 24 & 0.26 & ns & 0.40 & $<0.05$ \\
\hline \multirow{6}{*}{ (b) } & Radiotherapy & & & & & \\
\hline & Visual grading & 15 & $0 \cdot 21$ & ns & $0 \cdot 28$ & ns \\
\hline & $\begin{array}{l}\text { Mitotic index } \\
\text { Quantimet indices }\end{array}$ & 15 & $0 \cdot 23$ & ns & 0.20 & ns \\
\hline & 1 All values & 15 & 0.53 & $<0.05$ & 0.60 & $<0.05$ \\
\hline & 2 Values > 10000 & 14 & 0.52 & $<0.05$ & 0.61 & $<0.05$ \\
\hline & 3 Values > 15000 & 12 & 0.05 & ns & $0 \cdot 20$ & ns \\
\hline
\end{tabular}

with the Quantimet indices (Fig. 4), and there was some evidence that Quantimet measurements could rank the histologically more malignant tumours, for if indices over 10000 alone were considered a significant coefficient was still obtained, although this was not so with indices over 15000 .

(b) Patients receiving radiotherapy The same pattern of results was obtained with this group of patients. Grading by visual assessment correlated poorly with postoperative survival time, but Quantimet indices were significantly correlated both as a whole and when indices over 10000 were considered separately.

Examples of individual patients that were reclassified by Quantimet measurements to give the better correlation with postoperative survival time included: $(a)$ two patients with low indices (10 495 and 9245 ) who had been placed in visual grade 3 (Fig. 5); these were from the longest surviving patients in this grade since both were alive 54 and 60 months respectively after operation, $(b)$ one patient with an intermediate index of 23515 who had been placed in visual grade 4 , but the survival time of $\mathbf{4 0}$ months indicated that the neoplasm was not highly malignant, and $(c)$ one patient with an intermediate index of 19648 who had been placed in grade 2 (Fig. 6) but lived only seven months, suggesting that the neoplasm was not at the benign end of the spectrum of behaviour.

\section{Total duration of illness}

Comparison of the total duration of the illness with the two methods of histological grading gave similar results to those for postoperative survival time, but in all tests except mitotic index correlation was rather closer. Two differences were found in those patients who had not received radiotherapy: ( $a$ ) visual grading was now significantly correlated, and (b) Quantimet indices over 15000 now showed a significant correla- 


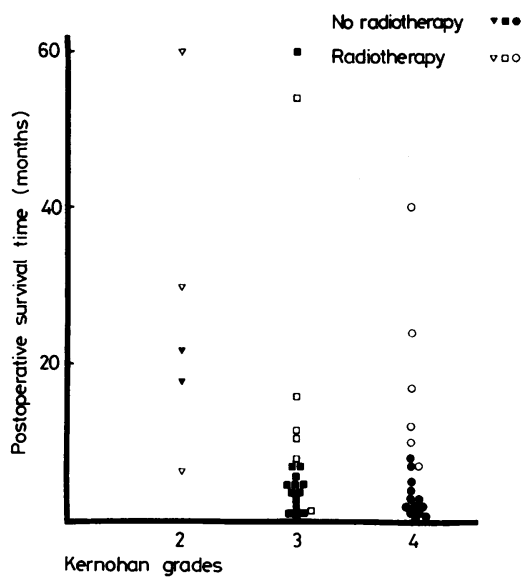

Fig. 3 Scatter diagram showing relationship between visual grading and postoperative survival time of 29 patients treated with surgery alone and 15 patients treated with combined surgery and radiotherapy.

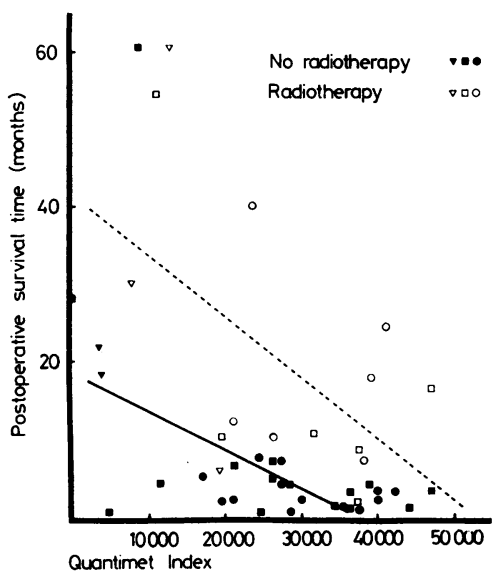

Fig. 4 Diagram showing relationship between Quantimet index and postoperative survival time of 29 patients treated with surgery alone and 15 patients treated with combined surgery and radiotherapy. Regression line for patients treated by surgery alone is solid line; regression line for patients receiving radiotherapy is interrupted line. Kernohan grade 2, $\nabla \nabla ;$ grade 3, $\square \square ;$ grade 4, $\bigcirc \bigcirc$.

tion, in addition to indices over 10000 and the values as a whole.

\section{Discussion}

Optical density/area profile measurements have not been used widely for histometry, because the sequential optical density threshold facility of the Quantimet was introduced only recently. Used with haemalum-stained sections, the method is empirical and does not measure any specific cytological or chemical tissue constituent. Haemalum staining includes all basophil tissue components although nuclei make the largest contribution. Changes in optical density/area profile reflect both the variation in the number of nuclei in a field and the quantity of staining of nuclei and other tissue constituents. Clearly, it is important to standardise the preparation of sections as far as possible, and for this reason Mayer's formula was chosen since this is a progressive stain and therefore avoids the process of differentiation which cannot be effectively controlled.

Comparison of visual grading with Quantimet indices showed that there were considerable differences, suggesting that the two methods were assessing different histological characteristics. Although there was some agreement about the selection of the less malignant neoplasms, the Quantimet apparently would place more into this group; there was no agreement about the ranking of the more highly malignant neoplasms.

In our hands, visual assessment was only moderately successful in separating the less malignant from the more highly malignant gliomas and was of no value for individual prognosis at the more common highly malignant end of the spectrum. This finding is supported by several previous studies which have shown that, in practice, surgical specimens of astrocytomas cannot usefully be separated into four grades described by Kernohan and Sayre (1952), and particularly that their grades 3 and 4 do not relate to survival time (Weir, 1973; Sherbet and Lakshmi, 1974; Stage and Stein, 1974; Weir and Grace, 1976).

By contrast, the Quantimet index derived from the optical density/area profile of neoplasms correlated well with the patients' survival time, and successfully ranked the gliomas. Correlation was sufficiently close to suggest that the Quantimet index could be used to give a reasonably accurate prognosis for the individual patient, which is not feasible by visual grading. Review of those neoplasms placed in too high a visual grade and apparently more correctly graded by the Quantimet suggested that ton much weight had been placed on easily observed visual features such as pleomorphism of nuclei, giant and multinucleate cells, and mitotic figures. The improved prediction obtained from optical density/area profile measurements indicated that more emphasis should have been put on cellularity (that is, the number of nuclei in a field relative to the internuclear material), a feature which is difficult to assess visually without point-counting. As previously 


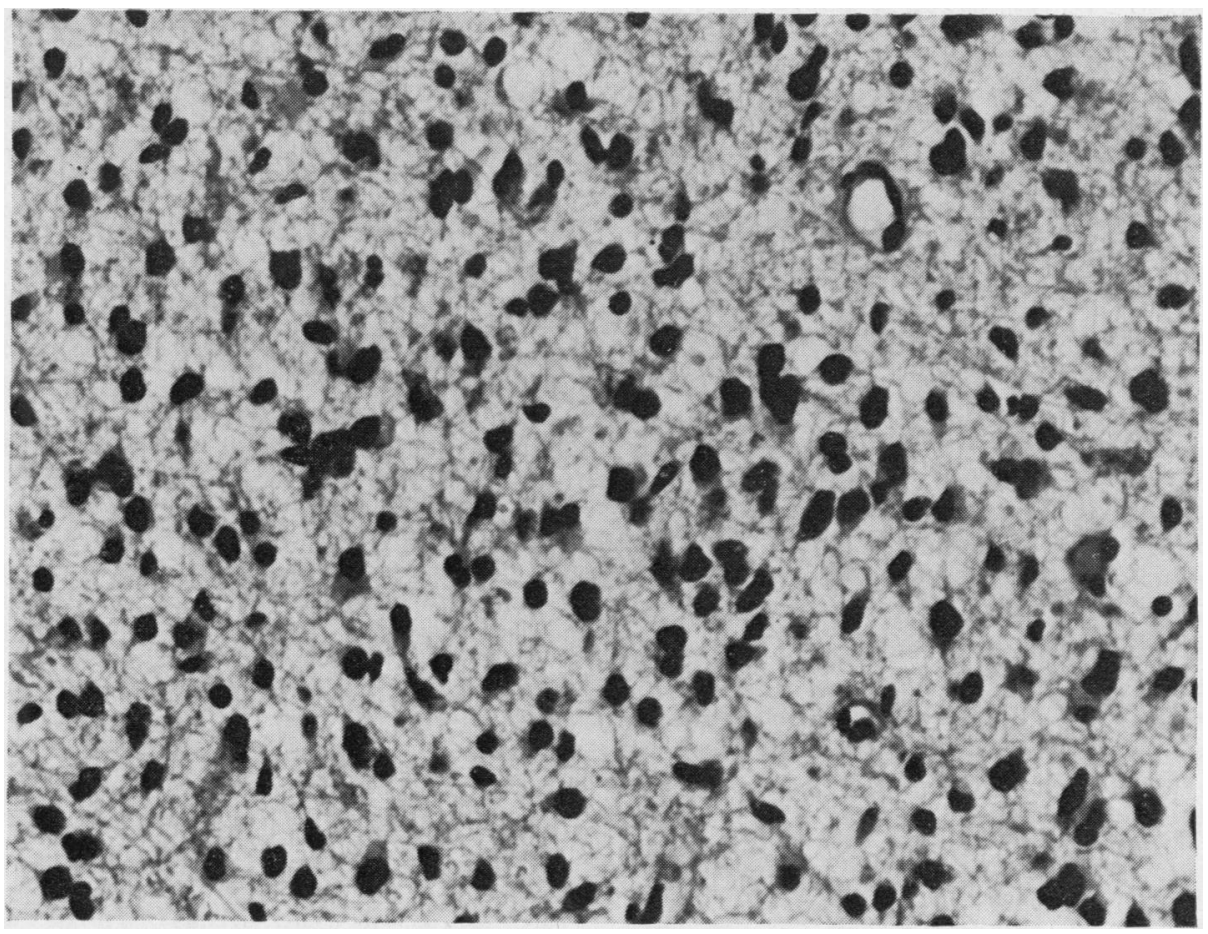

Fig. 5 Typical field of astrocytoma classified visually as grade 3. Quantimet index of 10495 suggested that malignancy of neoplasm was relatively low. Patient alive 54 months after operation. (Haematoxylin and eosin $\times 380$ )

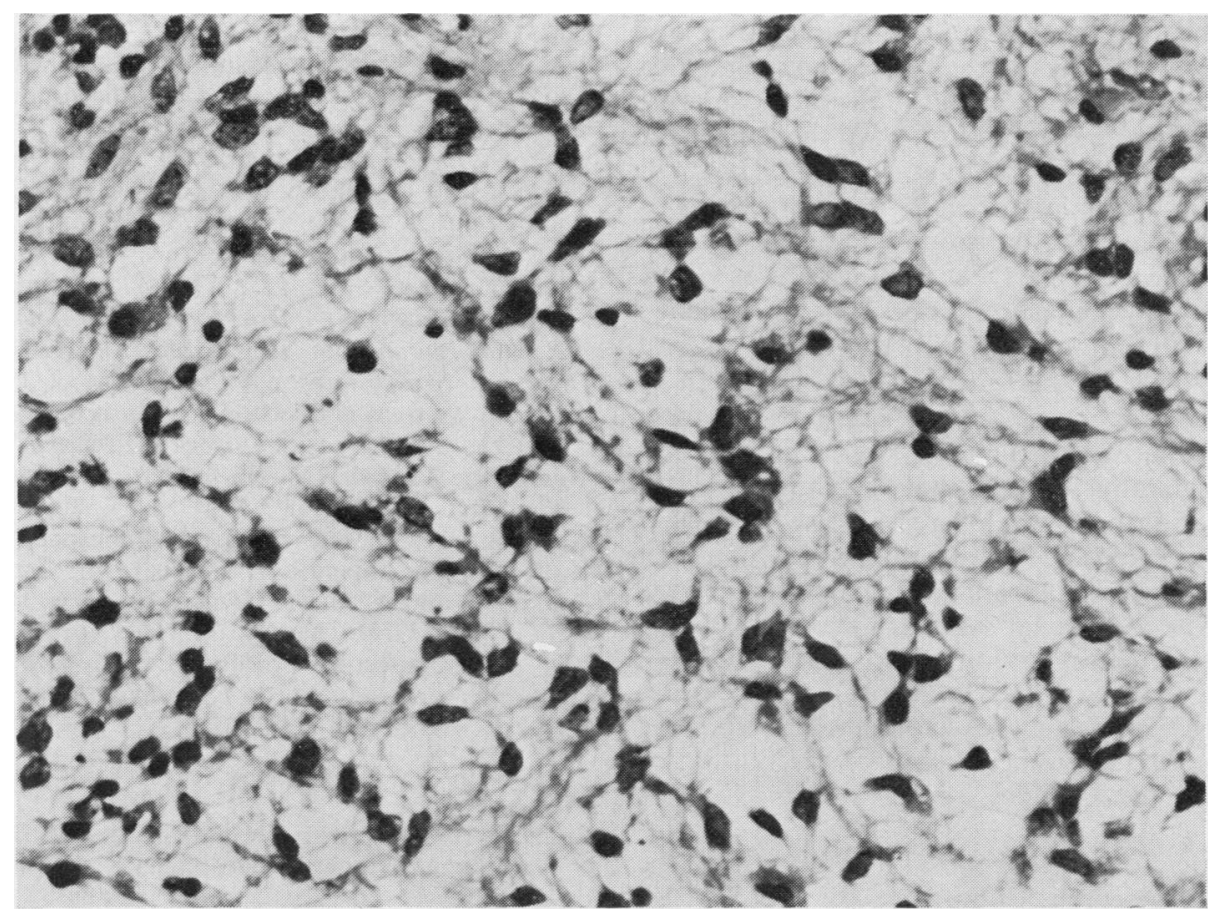

Fig. 6 Typical field of astrocytoma classified visually as grade 2. Quantimet index of 19648 suggested that malignancy of neoplasm was higher than visual grading inferred. Patient died seven months after operation. ( $H$ and $E \times 380$ ) 
reported by Frankel and German (1958), mitotic activity was of little predictive value, yet this is a feature to which pathologists traditionally give much importance.

The 15 patients in this series selected for radiotherapy were generally those without severe neurological deficit after operation and were on average younger and more often had received radical surgery than the remainder. Therefore no definite conclusions can be reached about the effects of radiotherapy, but ranking of these patients' tumours by Quantimet indices was consistent with the view that radiotherapy prolongs the survival time of patients with malignant gliomas (Frankel and German, 1958; Bouchard and Peirce, 1960; Roth and Elvidge, 1960; Bloor et al., 1962; Taveras et al., 1962; Weir, 1973). The Quantimet indices showed this rather more convincingly than visual grading, and in a larger series Quantimet ranking would certainly add precision to a study of the effects of radiotherapy on gliomas. At present, the value of radiotherapy for the less malignant astrocytomas is uncertain (Moss et al., 1973) but it is not yet known whether these neoplasms are unresponsive, or whether the inaccuracy of traditional histological grading methods has prevented adequate analysis of results of treatment.

One difficulty inherent in grading surgical biopsies of astrocytomas is sampling error (Russell and Rubinstein, 1977). It is well known that some astrocytomas show major structural differences from area to area, and this was certainly encountered in our series. However, we seem to have overcome this difficulty by selecting areas that in histological terms appear most malignant, although we recognise that anomalous results may be obtained from very small biopsies. For example, one small non-homogeneous biopsy was placed in visual grade 3 on the basis of the worst fields seen, but the Quantimet index was falsely low at 4668 since the total course of the patient's illness was nine months and postoperative survival one month (Fig. 4); this Quantimet error was due to a majority of the measurements being derived from tumour of relatively benign histological appearance. Overall our study suggests that the importance of sampling error has been overemphasised and that this is outweighed by the advantages of Quantimet measurement. These advantages include objectivity, which makes assessment less variable from day to day and less dependent on the experience of the pathologist, and sensitivity, which allows measurement over a wide scale, thus avoiding the perplexing problem of the neoplasm which is on the borderline between two visual grades.

This investigation was supported by a grant from the Equipment Research Committee of the Scottish
Home and Health Department. We thank Mr J. Block, Mr I. Jacobson, and Dr W. C. Swanson for $\underline{\text { o }}$ access to the clinical records.

\section{References}

Bloor, R. J., Templeton, A. W., and Quick, R. S. (1962). Radiation therapy in the treatment of intracranial 0 tumors. American Journal of Roentgenology, Radium Therapy and Nuclear Medicine, 87, 463-472.

Bond, C. P. (1976). Recognition of patterns in biological structures using multithreshold plots. (Abstract.) $\vec{\omega}$ Royal Microscopical Society Proceedings, 11 (5), Supplement, 52.

Bouchard, J., and Peirce, C. B. (1960). Radiation therapy in the management of neoplasms of the central nervous $\vec{i}$ system, with a special note in regard to children: twenty years' experience 1939-1958. American Journal of Roentgenology, Radium Therapy and Nuclear Medicine, 0 84, 610-628.

Frankel, S. A., and German, W. J. (1958). Glioblastoma multiforme: review of 219 cases with regard to natural history, pathology, diagnostic methods, and treatment.Journal of Neurosurgery, 15, 489-503.

Kernohan, J. W., and Sayre, G. P. (1952). Tumors of the Central Nervous System (Atlas of Tumor Pathology 1st $\square$ Series. Section 10, fasc. 35). Armed Forces Institute of Pathology, Washington.

Moss, W. T., Brand, W. N., and Battifora, H. (1973). Radiation Oncology, 4th edition. Mosby, St Louis.

Roth, J. G., and Elvidge, A. R. (1960). Glioblastoma multiforme: a clinical survey. Journal of Neurosurgery, $\overrightarrow{\overrightarrow{0}}$ 17, 736-750.

Russell, D. S., and Rubinstein, L. J. (1977). Pathology of Tumours of the Nervous System, 4th edition. Arnold, London.

Sherbet, G. V., and Lakshmi, M. S. (1974). Tumour grading by implantation in embryos. II. Grading of 0 some human astrocytomas. Journal of the National 3 Cancer Institute, 52, 687-692.

Stage, W. S., and Stein, J. J. (1974). Treatment of malig-윽 nant astrocytomas. American Journal of Roentgenology, Radium Therapy and Nuclear Medicine, 120, 7-18.

Taveras, J. M., Thompson, H. G., and Pool, J. L. (1962). D Should we treat glioblastoma multiforme? A study of survival in 425 cases. American Journal of Roentgenology, Radium Therapy and Nuclear Medicine, 87, 473-479.

Weir, B. (1973). The relative significance of factors $N$ affecting postoperative survival in astrocytomas $\omega$ grades 3 and 4. Journal of Neurosurgery, 38, 448-452.

Weir, B., and Grace, M. (1976). The relative significance of factors affecting postoperative survival in astro- $\mathbb{\complement}$ cytomas grades 1 and 2. Canadian Journal of Neuro-? logical Sciences, 3, 47-50.

Requests for reprints to: Dr J. M. Anderson, Department $\stackrel{\bar{Q}}{\varrho}$ of Pathology, Ninewells Hospital, Ninewells, Dundee DD2 1UB. 